\title{
Análisis de un algoritmo para la clasificación semiautomática de latidos en ECG
}

\author{
M. Llamedo Soria1,2,3, J.P. Martínez1,3

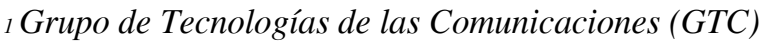 \\ Instituto de Investigación en Ingeniería de Aragón (I3A) \\ Universidad de Zaragoza, Mariano Esquillor s/n, 50018, Zaragoza, Spain. \\ Tel.+34-976762707, Fax+34-976762043, e-mail: llamedom@unizar.es \\ ${ }_{2}$ Departamento de Electrónica. \\ Universidad Tecnológica Nacional \\ Buenos Aires, Argentina \\ ${ }_{3}$ CIBER de Bioingeniería, Biomateriales y Nanomedicina (CIBER-BBN) \\ España
}

\begin{abstract}
Resumen En este trabajo se presenta un algoritmo para la clasificación de latidos en la señal de ECG, que puede operar tanto de manera automática como asistida. El mismo se compone por un clasificador automático previamente validado, y un algoritmo de clustering. Tanto el clasificador automático como el algoritmo de clustering utilizan características descrciptivas del ritmo de los latidos y de su morfología. Mediante la integración de las decisiones de ambos algoritmos, el algoritmo presentado puede desempeñarse de manera automática o con diversos grados de asistencia, dependiendo de la idoneidad del usuario. El algoritmo fue evaluado en la base de datos de arritmias del MIT-BIH con el propósito de comparar su rendimiento. En el modo automático de funcionamiento, el algoritmo propuesto ha obtenido un rendimiento ligeramente superior al clasificador automático original; pero con solo 5 latidos anotados manualmente en 22 registros, se ha obtenido una mejora del $5 \%$ en exactitud (A), sensibilidad $(S)$ y valor predictivo positivo $\left(P^{\wedge}\{+\}\right)$ globales. Para el modo completamente asistido, este algoritmo ha igualado el rendimiento de referencia con 55 veces menos esfuerzo manual y lo ha superado con 42. Estos resultados representan una mejora en el estado del arte, concluyendo que el rendimiento de un clasificador automático puede mejorarse mediante el uso eficiente de la ayuda provista por un experto.
\end{abstract}

